\title{
LINEAR DYNAMICAL SYSTEMS
}

\author{
E. FLYTZANIS
}

\begin{abstract}
We consider a probability measure $m$ on a Hilbert space $X$ and a bounded linear transformation on $X$ that preserves the measure. We characterize the linear dynamical systems $(X, m, T)$ for the cases where either $X$ is finite dimensional or $T$ is unitary and we give an example where $T$ is a weighted shift operator. We apply the results to the limit identification problem for a vector-valued ergodic theorem of A. Beck and J. T. Schwartz, $n^{-1}\left(\sum_{i}^{n} T^{i} F_{i}\right) \rightarrow \bar{F}$ a.s., where $F_{i}$ is a stationary sequence of integrable $X$ valued random variables and $T$ a unitary operator on $X$.
\end{abstract}

A dynamical system is defined as a triplet $(X, m, T)$, where $X$ is a separable metric space, $m$ is a probability measure on the Borel $\sigma$-algebra of $X$ and $T$ : $X \rightarrow X$ is a continuous measure preserving transformation (m.p.t.). If, in addition, $X$ is a linear topological vector space and $T$ a continuous linear transformation, then the dynamical system will be called linear. Linear dynamical systems can serve as models for representing stationary stochastic sequences as orbit sequences under $T$ of linear functionals on $X$. In this study we take $X$ to be a separable complex Hilbert space.

Definitions. (i) A complex number $\lambda$ is called an eigenvalue of the m.p.t. $T$ if there exists a complex valued measurable functions $f(\cdot)$ on $X$ such that $f(T(\cdot))=\lambda f(\cdot)$ a.e. It follows that the eigenvalues form a countable subgroup of the circle group. (ii) the m.p.t. $T$ is called ergodic if

$$
m\left[A \triangle T^{-1}(A)\right]=0
$$

implies $m(A)=0$ or 1 . If $T$ is ergodic then the eigenfunctions are constant a.e. in modulus, and any two corresponding to the same eigenvalue differ only by a multiplicative constant. (iii) The support of $m$ is defined as the collection of those points in $X$ whose every neighborhood has nonzero measure. It follows that the support is a closed subset of $X$, invariant under $T$, having measure 1. We can assume w.l.o.g. that the Hilbert space $X$ is spanned by the support of $m$.

In $\S 1$ we characterize the linear dynamical systems for the case where $X$ is finite dimensional. In $\$ 2$ we consider the infinite dimensional case but with $T$ restricted to being a unitary operator. This would include also the case where $T$ is totally bounded, i.e. $\left\|T^{n}\right\| \leqslant M<\infty, n=1,2, \ldots$ In $\S 3$ we consider an application to the limit identification problem for a vector-valued ergodic theorem. Finally we give a more general example of a linear dynamical system by using a weighted shift operator.

Received by the editors April 2, 1975.

AMS (MOS) subject classifications (1970). Primary 28A65; Secondary 47A10.

Key words and phrases. Measure preserving transformation, dynamical system, spectrum, linear operator Hilbert space measure, ergodic theorem, stationary stochastic process, shift operator. 
1. Finite dimensional case. Let $(X, m, T)$ be a linear dynamical system with $X n$-dimensional. By choosing a suitable base $\left(e_{i}\right), 1 \leqslant i \leqslant n$, we can represent $T$ as an $n \times n$ matrix $\left(a_{i j}\right)$ in the Jordan canonical form. We denote the coordinate functions by $f_{i}(\cdot)$.

THEOREM 1. With the above notation, $\left(a_{i j}\right)$ is a diagonal matrix whose eigenvalues considered as a linear operator in the Hilbert space $X$ are also eigenvalues of the m.p.t. T, considered as a m.p.t. in the measure space $X$. If the m.p.t. $T$ is also ergodic then the support of $m$ lies in an $n$-dimensional torus of the form: $\left\{x:\left|f_{i}(x)\right|=c_{i}, 1 \leqslant i \leqslant n\right\}$.

Proof. Suppose $\left(a_{i j}\right)$ is not diagonal. Then for some eigenvalue $\lambda$ of $\left(a_{i j}\right)$ there exist coordinate functions $f=f_{i-1}$ and $g=f_{i}$ such that for all $x \in X$,

(a) $f(T x)=\lambda f(x)+g(x)$,

(b) $g(T x)=\lambda g(x)$.

Since $X$ is spanned by the support of $m$, it follows that $g(\cdot)$ is not the zero function and $\lambda$ is an eigenvalue of the m.p.t. $T$, in particular, $|\lambda|=1$. By repeated applications of $T$ in the equations above and substitutions of (b) in (a) we obtain,

$$
f\left(T^{k} x\right)=\lambda^{k} f(x)+k \cdot \lambda^{k-1} g(x), \quad \forall k=1,2, \ldots, \text { all } x \in X .
$$

It follows that

$$
\left|f\left(T^{k} x\right)\right|=\left|k \cdot \lambda^{k-1} g(x)+\lambda^{k} f(x)\right| \geqslant k|g(x)|-|f(x)|, \quad \forall k=1,2, \ldots
$$

We can find $\varepsilon>0$ such that for $A=\{x:|g(x)|>\varepsilon\}$ we have $m(A)>0$, because we have assumed that $X$ is spanned by the support of $m$. Next we can find $c>0$ large enough so that for $B=\{x:|f(x)|<c\}$ we have $m(A \cap B)$ $>0$. We set $E=A \cap B$. Since $T$ is a m.p.t. on a finite measure space we can find an increasing sequence $k_{i}$ of positive integers such that $m\left(T^{-k_{i}} E \cap E\right)$ $>0$. However, the inequality gives $\left|f\left(T^{k} x\right)\right| \geqslant k \varepsilon-c>c$ for all $k$ sufficiently large independently of $x \in E$ and therefore $T^{-k} E \cap E$ is empty for all $k$ sufficiently large, a contradiction. Therefore $\left(a_{i j}\right)$ is diagonal. If, in addition, $T$ is ergodic, then the coordinate functions must be constant a.e. because they are eigenfunctions of the ergodic m.p.t. $T$. Q.E.D.

The above theorem characterizes completely the linear dynamical systems on a finite dimensional space. In particular, if $T$ is ergodic and $\lambda_{i}, 1 \leqslant i \leqslant n$, are the eigenvalues of the linear transformation, then the support of $m$ is topologically isomorphic to the closed subgroup generated by $\left(\lambda_{1}, \ldots, \lambda_{n}\right)$ of the multiplicative group of the $n$-dimensional torus. The transformation $T$ is given by multiplication by the generator and $m$ is the Haar measure on the subgroup.

2. Infinite dimensional case. Let $X$ be a separable complex Hilbert space and $(X, m, T)$ a linear dynamical system. We assume also that $T$ is unitary and make use of the following result from [2].

" $X$ is spanned by the eigenvectors of $T$ if there are no flight vectors of $T$. $x \in X$ is called a flight vector of $T$ if there is an increasing sequence $\{n\}$ of positive integers such that the sequence $T^{-n}(x)$ converges to zero weakly."

THEOREM 2. Let $(X, m, T)$ be a linear dynamical space in a separable Hilbert 
space and let $T$ be unitary. Then $X$ is spanned by the eigenvectors of $T$ and the eigenvalues of the linear operator $T$ are also eigenvalues of the m.p.t. T. If in addition the m.p.t. T. is ergodic, then the support of $m$ is contained in $a$ countable-dimensional torus.

Proof. Let $y$ be a flight vector of $T$, and let $y(\cdot)=(\cdot, y)$ be the linear functional defined by $y$. Since $X$ is spanned by the support of $m$, it follows that $y(\cdot)$ is not the zero function and we can find $c>0$ such that for $A=\{x$ : $|y(x)|<c\}$ we have $m(A)<1$. Let $\left\{n_{j}\right\}$ be the increasing sequence of positive integers for which $T^{-n_{j y}}(x)=y\left(T^{n_{j}} x\right) \rightarrow 0$, for all $x \in X$. We set $A_{j}=\left\{x:\left|y\left(T^{n_{j}} x\right)\right|<c\right\}$. We have clearly $\lim \inf \left\{A_{j}\right\}=X$ and therefore $\lim \inf \left\{m\left(A_{j}\right)\right\}=1$ by Fatou's lemma. We also have $T^{-n_{j}}\left(A_{j}\right) \subset A$ and therefore $m\left(A_{j}\right)=m\left(T^{-n_{j}} A_{j}\right) \leqslant m(A)<1$, a contradiction. Therefore the eigenvectors of $T \operatorname{span} X$. If $y$ is an eigenvector of $T$ corresponding to the eigenvalue $\lambda$, then $y(\cdot)=(\cdot, y)$ is an eigenfunction of the m.p.t. $T$ with eigenvalue $\lambda$. If $T$ is also ergodic then the support of $m$ is contained in a cylinder set of the form $|y(\cdot)|=c$. Taking now a basis of eigenvectors, we obtain the last statement of the theorem. Q.E.D.

This theorem characterizes completely the linear dynamical systems that can be induced in a Hilbert space by a unitary operator.

3. Vector-valued ergodic theorem [1]. Let $(S, \Sigma, m)$ be a probability measure space and $h: S \rightarrow S$ a m.p.t. Let $X$ be a separable, reflexive Banach space and $F: S \rightarrow X$ an integrable vector valued function. $T$ is a bounded linear operator on $X$ with $\|T\| \leqslant 1$. Then there is an integrable $\bar{F}: S \rightarrow X$ such that

$$
\lim \left[n^{-1} \sum_{1}^{n} T^{i} F\left[h^{i}(s)\right]\right]=\bar{F}(s), \quad \text { a.e. in } S,
$$

strongly in $X$ and also in the mean of order $1 . \bar{F}$ satisfies the equation $\bar{F}(s)=T \bar{F}[h(s)]$ a.e. in $S$.

Taking $X$ to be a Hilbert space and $T$ a unitary operator we note that the function $\bar{F}: S \rightarrow X$ induces on $X$ a measure which is invariant under $T$. It follows that in the space $\bar{X}$ spanned by the support of the induced measure, which coincides with the essential range of $\bar{F}$, the unitary operator $T$ has complete point spectrum, and its eigenvalues are also eigenvalues of the m.p.t. $h$. The solution $\bar{F}$ is now obtained as follows: Let $\lambda_{i}, i=1,2, \ldots$, be the eigenvalues common to both $h$ and $T$ and $P_{i}$ the projections on the corresponding eigenspaces of $T$. We set $\bar{F}_{i}=P_{i} \bar{F}$. It is clearly sufficient to identify $\bar{F}_{i}$, because then $\bar{F}=\sum \bar{F}_{i}$. We set also $F_{i}=P_{i} F$. Then $\bar{F}_{i}$ is the limit, as given by the ergodic theorem, of the sequence

$$
n^{-1} \sum_{k=1}^{n} T^{k} F_{i}\left[h^{k}(\cdot)\right]=n^{-1} \sum_{k=1}^{n} \lambda_{i}{ }^{k} F_{i}\left[h^{k}(\cdot)\right] .
$$

These limits are easily obtained. In particular: (i) if $h$ is ergodic and $f_{i}(s)$ are the eigenfunctions of $h$ corresponding to the eigenvalues $\lambda_{i}$ then

$$
\bar{F}=\sum \bar{f}_{i}(s) x_{i}, \quad \text { a.e., where } x_{i}=\int F_{i}(s) \cdot f_{i}(s) m(d s) \in X .
$$

(ii) If $h$ and $T$ have no eigenvalues in common expect possibly the number 1 and $P=\lim \left(n^{-1} \sum_{1}^{n} T^{i}\right)$ is the projection on this eigenspace, then $\bar{F}$ 
$=E\left(P F / \Sigma_{0}\right)=$ expected value with respect to the sub- $\sigma$-field $\Sigma_{0}$ generated by the sets in $\Sigma$ that are invariant under $h$.

4. Conclusions. We note that the class of dynamical systems that can be represented as linear ones is very restricted if either the space is finite dimensional or the operator is unitary, in fact they are exactly the systems having complete point spectrum. However, a more general theory is needed, as indicated by the following construction.

ExAmple. We consider the backward shift in the space of one-sided sequences square-summable with respect to a weight. Let $\{r(n)\}, n=0$, $1, \ldots$, be a weight sequence satisfying the conditions

(1) $\sum r(n)<\infty$,

(2) $\sup _{n}[r(n) / r(n+1)]<\infty$.

Let $l^{2}(r)$ be the Hilbert space of all one-sided sequences $\left\{a_{n}\right\}$ for which the norm $\|a\|=\left[\Sigma\left|a_{n}\right|^{2} r(n)\right]^{1 / 2}$ is finite. The shift operator $T$ on $l^{2}(r)$ is defined by $T\left(a_{0}, a_{1}, \cdots\right)=\left(a_{1}, a_{2}, a_{3}, \cdots\right)$. The operator $T$ is bounded and

$$
\|T\|=\sup [r(n) / r(n+1)]^{1 / 2} \quad(\operatorname{cf} .[3, \text { Chapter 10]). }
$$

We consider also any m.p.t. $h$ on a probability space $(S, \Sigma, m)$ and $f(\cdot)$ a square integrable complex valued function on $S$. Then the expression $F(\cdot)$ $=\left[f(\cdot), f[h(\cdot)], f\left[h^{2}(\cdot)\right], \cdots\right]$ defines an $l^{2}(r)$-valued function a.e. on $S$ because of (1). This function induces a probability measure on $l^{2}(r)$ which is invariant under $T$ because

$$
F[h(\cdot)]=\left[f[h(\cdot)], f\left[h^{2}(\cdot)\right], \cdots\right]=T F(\cdot) \text { a.e. }
$$

We can also consider a two-sided shift if $h$ is invertible. The example is certainly not covered by the theory above as $h$ may not have any eigenvalues.

\section{REFERENCES}

1. A. Beck and J. T. Schwartz, A vector-valued ergodic theorem, Proc. Amer. Math. Soc. 8 (1957), 1049-1059. MR 20 \#624.

2. K. Jacobs, Lecture notes on ergodic theory. Parts I, II, Mathematisk Institut, Aarhus Universitet, Aarhus, 1963. MR 28 \#138; erratum, 28, p. 1247.

3. P. R. Halmos, A Hilbert space problem book, Van Nostrand, Princeton, N. J., 1967. MR 34 \#8178.

Polytechnic School, University of Thessaloniki, Thessaloniki, Greece 